\title{
L'organisation de la radioprotection professionnelle à Electricité de France
}

\author{
P. HALLER* \\ (Manuscrit reçu le 20 janvier 1987)
}

\begin{abstract}
RÉSUMÉ
L'organisation actuelle de la radioprotection professionnelle à Electricité de France repose sur un ensemble de principes qui, au-delà du respect de la réglementation, visent à intégrer cette protection dans la conception des installations et la préparation du travail. Cette démarche s'appuie sur une analyse permanente du retour d'expérience afin d'aider les différents services concernés à mettre en œuvre les actions correctrices qui s'avèrent efficaces.

L'auteur présente les principales composantes autour desquelles se structure le système de la radioprotection des travailleurs: la doctrine, les différents services compétents, ainsi que les domaines d'intervention en vue de réduire les expositions.
\end{abstract}

\section{ABSTRACT}

The present organization of radiological protection at Electricite de France (the public corporation operating French nuclear power stations) is lying on basic principles which, beyond the respect of legal constraints, aim at integrating the protection of workers in the design of equipements and the preparation of operation and maintenance works. This approach is closely related to a systematic analysis of past experience to help the various services concerned to put into practice the most effective improving and corrective actions.

The author presents the main elements structuring the occupational radiological protection system at EdF : the doctrine, the different services involved as well as the various actions undertaken to reduce exposures.

\section{INTRODUCTION}

A la fin de l'année 1986, 33 tranches à réacteur à eau pressurisée (REP) de $900 \mathrm{MW}$ et 6 tranches de $1300 \mathrm{MW}$ étaient couplées au réseau d'Electricité de France; $95 \%$ de l'électricité nucléaire produite provient de la filière REP. La première tranche de $900 \mathrm{MW}$ a été couplée en avril 1977;

* EdF, Division Securité Radioprotection Environnement, 6, rue Ampère, BP 114, 93203 Saint-Denis Cedex 1 .

RADIOPROTECTION, VOL. $22 \cdot 0033-8451 / 1987 / 193 / \$ 5.00 /$ (C) Gédim. 
le rythme moyen de mise en service sur les dernières années se situe donc entre 4 et 5 tranches par an. Dans cette période, la puissance nucléaire installée est passée de 2275 à $43000 \mathrm{MW}$ et la production nucléaire de $12725 \mathrm{GWh} / \mathrm{an}$ à $237400 \mathrm{GWh} / \mathrm{an}$. La figure 1 représente la situation des centrales 900 et $1300 \mathrm{MW}$ au $1^{\mathrm{er}}$ janvier 1987. En 1985, l'énergie nucléaire

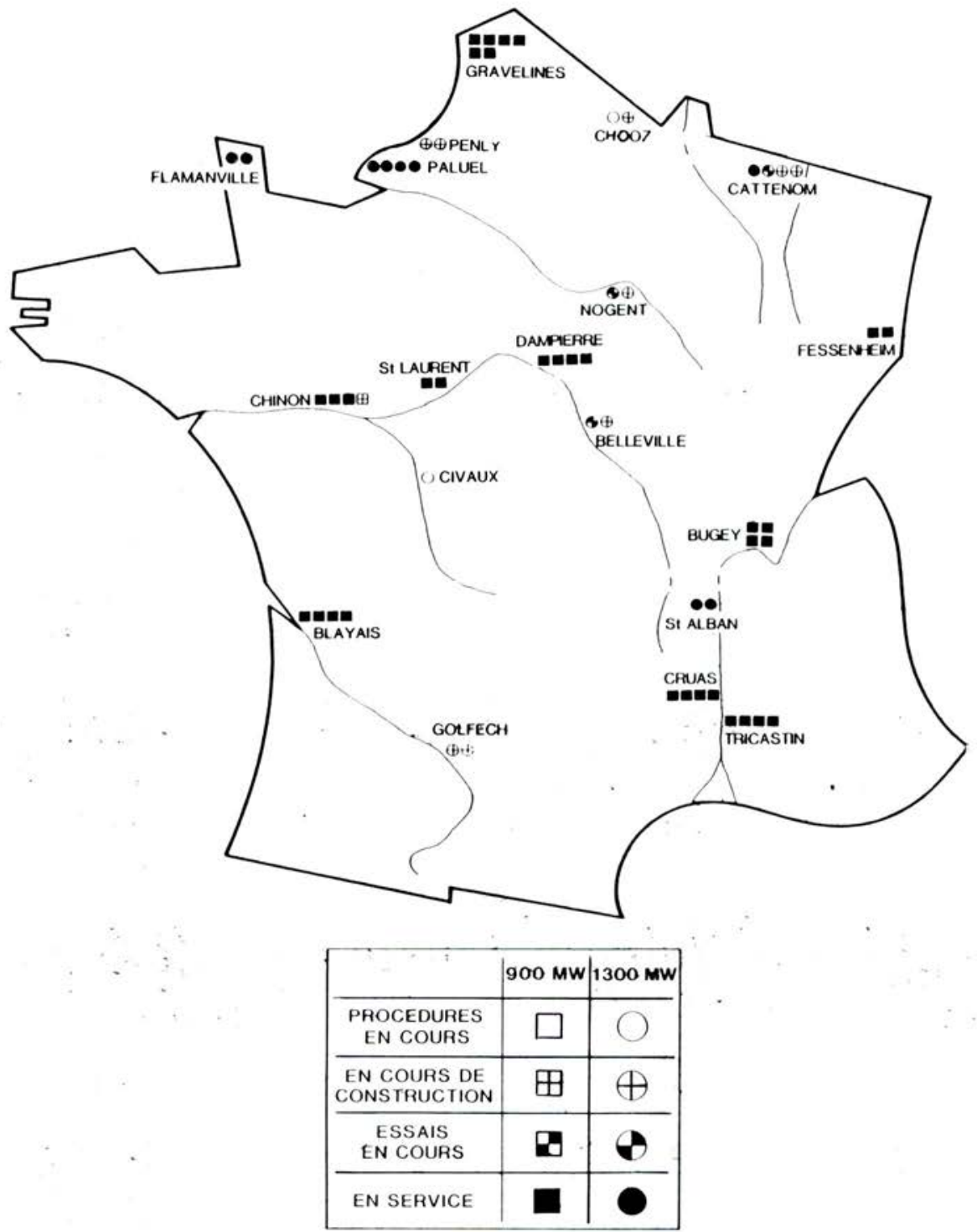

Fig. 1. - Sites nucléaires en janvier 1987 (REP). 
a contribué pour près de $70 \%$ à la production totale d'électricité française. Cette évolution de la puissance nucléaire installée s'est accompagnée d'une croissance importante du personnel directement affecté aux travaux sous rayonnements (DATR).

La figure 2 représente l'évolution du nombre d'agents DATR appartenant au personnel d'EdF. De 1000 en 1975, ce nombre est passé à plus de 12000 en 1986. Ce chiffre comporte essentiellement les agents travaillant dans les centrales. L'évolution du nombre d'agents DATR appartenant aux entreprises prestataires intervenant dans les centrales est tout à fait semblable, ces agents d'entreprises sont au nombre de 15000 environ à l'heure actuelle. La qualification et les tâches professionnelles confiées à ces agents recouvrent l'ensemble de la gamme habituellement rencontrée dans le milieu industriel: des manœuvres, des spécialistes et des ingénieurs. La moyenne d'âge des agents EdF en centrale nucléaire est inférieure à 30 ans. La majeure partie de ces agents possède une expérience récente de la radioprotection.

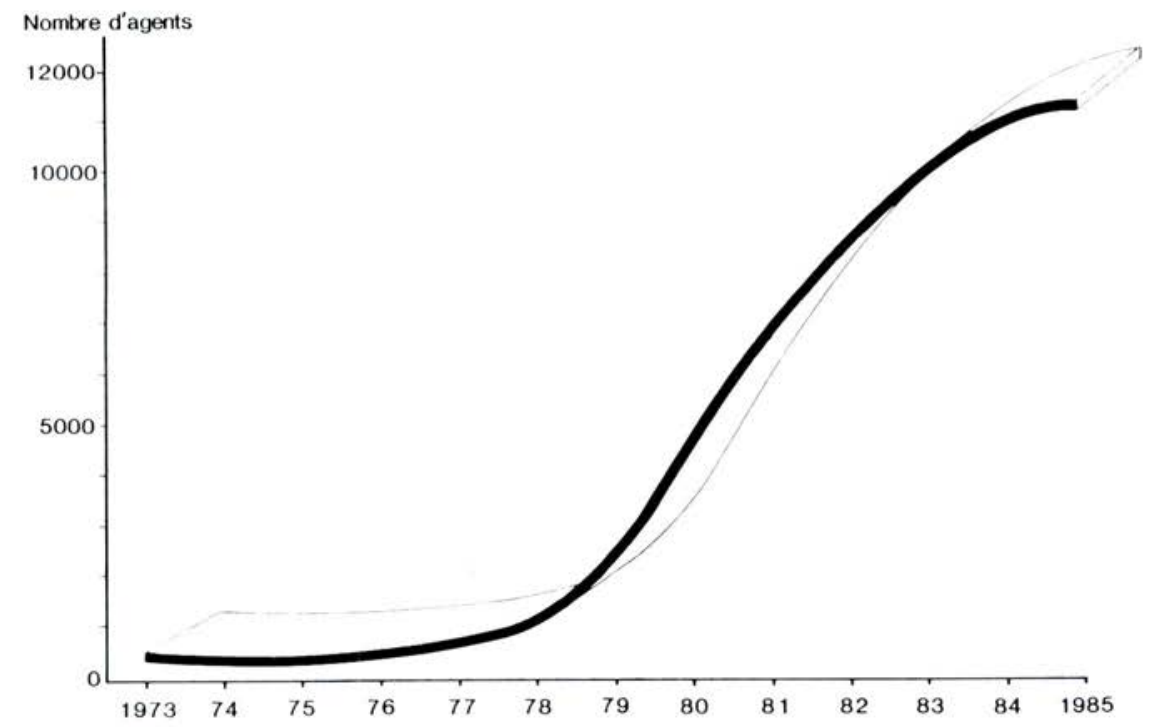

Fig. 2. - Effectif des agents directement affectés aux travaux sous rayonnements.

Le système de radioprotection des travailleurs doit donc tenir compte à la fois de cette évolution rapide de la technologie, des besoins de retour d'expérience liés à cette évolution, des besoins importants en personnel à former ainsi que des exigences industrielles et économiques. 


\section{LES COMPOSANTES DE LA RADIOPROTECTION}

Le système de la radioprotection appliqué au sein d'Electricité de France s'articule autour des axes principaux suivants :

- une doctrine de base qui précise les objectifs et les modalités d'application de la réglementation;

- une organisation qui représente la structure garantissant la mise en $œ u v r e$ et la pérennité de la doctrine;

- des domaines propres comportant les techniques, les matériels, les méthodes de travail contribuant à prévenir les risques radiologiques;

- enfin, la motivation indispensable des acteurs à la radioprotection. Elle se traduit par une éducation des mentalités à la prévention des risques.

C'est l'interaction harmonieuse et la non séparation de ces composantes qui garantissent l'efficacité du système de radioprotection.

Le présent exposé présentera les actions mises en œuvre au sein d'EdF au niveau de chacune de ces composantes.

\section{LA DOCTRINE EN MATIĖRE DE RADIOPROTECTION}

Le principe de base est évidemment le respect de la réglementation française en matière de protection des travailleurs contre les rayonnements ionisants [9]. Cette réglementation, qu'il ne convient pas d'expliciter ici, impose notamment le respect des limites de doses d'exposition individuelle ainsi que l'application du principe des doses les plus faibles possibles.

A EdF, la radioprotection est inscrite dans les objectifs généraux de prévention des risques professionnels, elle n'est pas séparée de la sécurité dite classique. A ce titre, les principes d'application sont identiques et les acteurs chargés de la mise en œuvre sont les mêmes $[5,7,11]$.

La radioprotection revêt un aspect de prévention collective et un aspect individuel. La sécurité-radioprotection est l'affaire de tous et de chacun. La radioprotection ne relève pas uniquement de la compétence de spécialistes. Le rôle de ces derniers est de garantir l'application des règles de radioprotection.

L'objectif est d'intégrer la radioprotection dans l'organisation de l'établissement, dès la conception des installations, puis dans la préparation du travail, bref, dans le réflexe quotidien de chacun à tous les niveaux hiérarchiques.

Cette intégration de la radioprotection n'est possible que si elle s'appuie sur la connaissance de la réalité du terrain où elle doit s'appliquer. C'est pourquoi le retour d'expérience est un élément essentiel d'une prévention efficace. II permet de restituer les informations aux différents services concernés. 


\section{L'ORGANISATION DE LA RADIOPROTECTION AU SEIN D'EDF}

Les centres de production nucléaire (CPN), comprenant jusqu'à 6 tranches, dépendent du Service de la production thermique lui-même rattaché à la direction de la production et du transport d'EdF.

La radioprotection est prise en compte aux niveaux d'une part des services centraux situés à Paris et d'autre part des CPN.

Au niveau national, il s'agit :

- du comité de radioprotection (CR);

- du service général de la médecine du travail (SGMT);

- du service "Prévention et sécurité" (SPS);

- du département Sécurité, radioprotection, environnement (DSRE).

Le CR, SGMT, SPS sont communs à l'ensemble des directions d'EdF. Le DSRE dépend du service de la production thermique.

\section{Comité de radioprotection (CR)}

Ce comité, composé de personnalités du milieu médical et de responsables de haut niveau de l'établissement EdF, est l'instance supérieure en matière de réglementation interne en radioprotection. Sa mission est:

- d'étudier et proposer les règles à appliquer à EdF;

- d'assurer, avec les organismes officiels et les personnalités ayant compétence en matière de radioprotection, toutes les liaisons destinées à faire connaître les positions d'EdF;

- d'émettre des avis sur les orientations à prendre.

Ce comité est présidé actuellement par $M$. le Prof. TUBIANA.

\section{Service général de la médecine du travail}

Ce service, dirigé par un médecin-chef du travail, est chargé de faire observer une unicité de doctrine à EdF-GdF dans l'organisation générale, l'équipement et le fonctionnement des services médicaux du travail.

Un médecin chargé de la radioprotection est attaché au médecin-chef. Un laboratoire d'analyses médicales (LAM) situé à Saint-Denis est spécialisé en analyses radiotoxicologiques.

\section{Service Prévention-sécurité (SPS) $[5,6]$}

Ce service, outre sa mission générale de prévention et de sécurité au sein d'EdF, est garant de la loi en matière de radioprotection. II participe aux travaux du comité de radioprotection. En liaison étroite avec le service de la production thermique, le SPS participe à l'élaboration de documents prescriptifs et à la formation des ingénieurs en radioprotection. Par ailleurs, le SPS est le représentant d'EdF auprès des ministères pour les questions réglementaires. 


\section{Le département "Sécurité - radioprotection - environnement" (DSRE)}

Ce département est placé sous l'autorité du sous-directeur, chef adjoint du service de la production thermique. Le département est composé de trois divisions dont deux s'occupent directement de la radioprotection des travailleurs. II s'agit de la division liaison-conception et de la division sécurité-radioprotection qui regroupent 28 ingénieurs et agents techniques.

Son action se situe dans une perspective de diminution des doses au niveau de la conception des installations, de la réduction de l'activité des circuits, de la saisie des conditions radiologiques d'intervention. Cette saisie des doses collectives, des localisations des débits de doses et des contaminations permet d'évaluer les risques et d'apporter les mesures correctives.

Ces actions sont complétées d'une politique volontariste de "promotion de la prévention" qui consiste à :

- recueillir et rediffuser l'information auprès des centrales, grâce à des contacts fréquents;

- évaluer les risques professionnels devant lesquels se trouve le personnel;

- définir les règles ou recommandations, les prescriptions de portée générale et les valider;

- définir et adapter les programmes de formation;

- proposer des méthodes et des moyens pratiques relatifs aux matériels de mesure et de protection individuelles et collectives;

- contrôler, par le retour de l'expérience, la validité des méthodes et leur apporter les correctifs nécessaires;

- prospecter, auprès d'organismes spécialisés, les moyens de prévention pouvant intéresser les activités du service.

Le DSRE assure, en outre sous contrôle du SCPRI (ministère de la santé), la dosimétrie réglementaire des agents EdF. Environ 17000 films sont traités chaque mois dans un atelier disposant de robots industriels et de machines automatisées. La tenue à jour des fichiers des agents DATR est réalisée conjointement par les centrales nucléaires et le DSRE, à l'aide d'un réseau de transmission de données informatiques vers l'ordinateur central IBM 3090-200 du service de traitement de l'information d'EdF.

Les études et analyses radiologiques sont réalisées soit dans les laboratoires des centrales nucléaires, soit confiées à des organismes spécialisés tels que le CEA.

\section{Organisation de la radioprotection dans les centres de production nucléaire (CPN)}

Un CPN est constitué d'une direction et de sous-unités correspondant chacune à une paire de tranches, et d'une sous-unité technique (SUT) chargée des travaux d'arrêts annuels. 
La responsabilité de la radioprotection est confiée aux chefs adjoints de ces sous-unités. Chacun est aidé dans sa tâche par une section sécuritéradioprotection (SRP) placée directement sous ses ordres et dirigée par un ingénieur. Une section SRP d'une paire de tranche comporte environ 10 personnes, celle d'une SUT environ 15 personnes.

Certains services ont leur part de responsabilité dans le domaine de la radioprotection:

- le service "conduite" doit veiller par des dispositions de circuits appropriés à réduire les doses collectives (filtration de circuit, permutation de filtres en temps utile, etc.);

- le service technique, et notamment la section chimie, doit minimiser par ses actions les problèmes de corrosion et suivre tout particulièrement les phénomènes d'activation des produits utilisés pour obtenir et maintenir la qualité du fluide primaire;

- le service "matériel", dans ses interventions d'entretien, doit être attentif aux moyens, aux choix des méthodes, aux modifications de matériels à envisager pour réduire à des valeurs aussi faibles que possible les doses nécessaires à l'entretien et l'exploitation des centrales.

\section{La section "Sécurité-radioprotection"}

La section "Sécurité-radioprotection" n'est pas un service chargé d'assurer toutes les tâches ayant un rapport avec la sécurité et la radioprotection. Elle n'est pas chargée de surveiller les intervenants en permanence. Ceci serait contraire à l'esprit général qui veut que tous les agents soient formés et se sentent concernés dans le domaine de la sécurité. En effet, si cette façon de faire peut avoir de réels mérites à court terme, elle ne permettrait pas d'obtenir une bonne efficacité pour la réduction des doses collectives à long terme.

Le rôle de la section peut être résumé par les mots :

ASSISTANCE, CONSEIL, CONTRÔLE, FORMATION, ANIMATION

- assistance technique pour le choix et la maintenance du matériel de radioprotection;

- conseil aux préparateurs pour l'élaboration des procédures d'interventions;

- conseil aux chefs de travaux sur les chantiers;

- contrôle des niveaux d'irradiation et de contamination; les mesures radiochimiques sont réalisées par le laboratoire du service technique;

- contrôle des dérives en matière de comportements et de savoirfaire;

- formation des agents;

- animation par une présence effective sur le terrain, collecte d'informations. 


\section{L'ingénieur "Sécurité-radioprotection" d'un CPN}

Un ingénieur assiste et conseille la direction d'un centre de production nucléaire. En particulier, il est chargé de suivre l'évolution et de se porter garant de la doctrine et de la politique définie par la direction du service de la production thermique.

\section{Le service de la médecine du travail}

La médecine du travail, à l'échelon du CPN, assure en ce qui concerne la radioprotection :

- l'organisation de la surveillance médicale du personnel et la tenue à jour des dossiers médicaux nécessaires à l'aptitude DATR des agents;

- la surveillance sur le plan de la contamination interne du personnel de la centrale;

- le traitement des cas de contamination externe supposant d'autres actions que le simple lavage ou comportant un risque de contamination interne;

- l'évaluation des effets des irradiations externes ou des contaminations internes incidentelles dépassant les limites admissibles et, dans les cas les justifiant, le traitement d'urgence et l'évacuation vers un centre spécialisé.

\section{La représentation du personnel}

Le comité d'hygiène, de sécurité et de conditions de travail (CHSCT) institué par la loi, est un organisme réunissant, au moins une fois par trimestre, la direction du centre de production nucléaire, la médecine du travail et des représentants du personnel pour évaluer les conditions d'hygiène, de sécurité et de travail. Sa compétence s'exerce également dans le domaine de la radioprotection.

II est à noter l'existence d'un comité national d'hygiène, de sécurité et de conditions de travail (CNHSCT) dont la compétence s'exerce sur l'ensemble d'EdF.

Les représentants du personnel pourraient également s'exprimer, en cas de besoin, sur des questions de radioprotection dans les comités mixtes à la production (CMP).

\section{LES DOMAINES DE LA RADIOPROTECTION}

Les domaines touchés par la réalisation des objectifs de réduction des doses définis par la doctrine sont les suivants:

- la conception des installations;

- la réduction de la radioactivité des circuits;

- la formation du personnel;

- l'accueil des entreprises prestataires; 
- la préparation du travail;

- le choix des matériels et des techniques de prévention.

\section{Conception des installations}

La conception des installations a été définie par la direction de l'équipement et notamment le SEPTEN de manière à minimiser les doses des intervenants.

De plus, le retour d'expérience permet d'apporter des modifications à la conception d'origine, dans une perspective de réduction des doses. A cet égard, on peut citer la mise en place de protections biologiques et de rampes de rinçage des piscines pour simplifier les opérations de nettoyage coûteuses en dose, le remplacement de l'acier des grilles des éléments combustibles par du zircalloy, l'amélioration des dispositifs de changement de cartouches des filtres très irradiantes.

\section{Réduction de la radioactivité des circuits}

La redéposition dans les tuyauteries des produits activés véhiculés par l'eau du circuit primaire et les effluents est une source importante d'irradiation du personnel. Des procédures de conditionnement de l'eau du circuit primaire ont été élaborées en collaboration avec le Commissariat à l'énergie atomique pour limiter cette redéposition [1].

\section{La formation du personnel [8]}

Un programme de formation diversifié a été mis au point, il comprend:

- des actions centralisées, sous forme de stages intensifs de durée d'une à deux semaines destinés aux:

- ingénieurs (formation générale et pluridisciplinaire),

- techniciens du contrôle technique (problèmes de mesures),

- techniciens de sécurité - radioprotection (animation - assistance),

- formateurs (réalisation des actions locales de formation);

- des actions décentralisées de démultiplication menées par les formateurs indiqués ci-dessus:

- premier palier (tous exécutants),

- deuxième palier (tous chefs de travaux).

A chacun de ces stages correspond un dossier pédagogique destiné à garantir l'homogénéité des formations délivrées. Ces dossiers, qui ont d'abord été essentiellement un effort de vulgarisation de la "science de la radioprotection", ont fait l'objet d'un suivi et de nombreuses mises à jour, devenant ainsi de plus en plus dépouillés de leurs aspects théoriques, et adaptés aux situations concrètes que les agents pourraient être amenés à rencontrer. 
Une action de réforme de la formation est en cours actuellement, de nouvelles orientations sont prises concernant l'intégration de la sécurité classique et de la radioprotection dans une démarche globale de prévention des risques professionnels, l'accent est porté sur la motivation du personnel.

Les formateurs sont autant que possible des hommes de terrain capables de transmettre l'expérience vécue. L'intervention de cadres de haut niveau valorise ces stages et, si besoin était, remotive ces intervenants euxmêmes en matière de sécurité-radioprotection.

Ce dispositif de formation a été complété par l'édition d'un certain nombre de documents pédagogiques, très largement diffusés, écrits ou audiovisuels. Des programmes de recyclage, sous forme d'enseignement assisté par ordinateur, ont été élaborés et font actuellement l'objet d'expériences encourageantes.

Un guide pratique de la radioprotection est mis à jour et diffusé chaque année. [7].

\section{Accueil des entreprises prestataires [3, 4]}

A chaque arrêt de tranche pour rechargement du combustible, un personnel important de différents constructeurs et prestataires est amené à intervenir pour réaliser des modifications ou de gros travaux d'entretien non réalisables par le personnel de la centrale.

II n'aurait pas été possible au chef de centrale d'assumer correctement sa responsabilité légale de chef d'établissement sans garantir que ce personnel d'entreprise possède une formation convenablement adaptée, lui permettant de s'intégrer harmonieusement dans l'organisation mise en place.

En vue de garantir une homogénéité de formation, EdF a mis à la disposition des entreprises les dossiers de formation pédagogique, en tous points identiques à ceux utilisés pour les agents EdF. Des rencontres périodiques entre des responsables EdF et les formateurs de ces entreprises sont organisées pour faire le point des difficultés rencontrées.

En contrepartie, il est demandé aux agents d'entreprises, lorsqu'ils se présentent sur un site, d'être porteurs d'un "carnet individuel de travailleur DATR" portant toutes les indications permettant de s'assurer de la régularité de leur situation; le chef de centrale se réserve le droit de faire effectuer des contrôles de connaissances, et éventuellement de refuser l'accès en zone contrôlée si ces contrôles s'avéraient non satisfaisants.

A I'heure actuelle, on peut estimer qu'environ 15000 agents d'entreprises ont bénéficié d'une formation en radioprotection comparable à celle des agents EdF.

\section{La préparation du travail}

La radioprotection doit être intégrée dans le travail à tous les niveaux, à commencer par l'habilitation des intervenants. Le titre d'habilitation RP1 ou 
RP2, signifiant que l'intervenant a atteint un niveau de formation en radioprotection appropriée, est indispensable à l'exécution du travail en zone contrôlée.

La préparation des interventions est confiée à un préparateur, il est chargé de penser la radioprotection avant les interventions et de l'intégrer dans les gammes d'interventions. II s'agit de définir les précautions pour réduire les doses, de confiner la contamination, d'assurer la bonne information du chef de travaux, et de garantir le retour d'expérience.

Le chef de travaux, quant à lui, assure la direction effective des travaux, il est responsable de la radioprotection et de son chantier en s'appuyant sur une procédure écrite fournie par le préparateur. II doit notamment contrôler le niveau d'irradiation ou de contamination régnant dans le local où doit se dérouler l'intervention, respecter les limites de doses à ne pas dépasser, vérifier que les dispositifs de protections contre la contamination sont utilisés conformément aux consignes. II doit veiller à l'état du chantier, à l'emballage et à l'évacuation des déchets radioactifs après contrôle. Dans les interventions particulières, le chef de travaux peut obtenir l'assistance de la section "Sécurité-radioprotection".

\section{Les moyens techniques de prévention}

Un grand nombre de techniques de prévention du risque radiologique sont devenues classiques, bien que certaines améliorations soient toujours en cours, pour les tenues étanches, les masques respiratoires, les protections biologiques, les enceintes de confinement, etc. II convient de souligner quelques aspects particuliers de ces moyens techniques.

Le dosimètre électronique à affichage numérique est porté par chaque agent en zone contrôlée en plus du film réglementaire. II permet de réaliser une dosimétrie journalière et d'effectuer les bilans dosimétriques par type de travail et par catégorie de personnel. Après traitement par ordinateur, il permet les retours d'expérience exploités à l'échelon local et national. Son système de clignotement (une fois par $0,1 \mathrm{mrem}$ ) en fait un "sixième sens" permettant de percevoir le débit de dose en temps réel. Les fabricants de ces dosimètres sont Merlin Gerin Provence et Saphymo-Stel.

Le confinement dynamique de la contamination est une technique qui est préconisée autant que possible à la place des enceintes de confinement dont le montage et l'évacuation sont pénalisants en temps et coûteux en doses.

Le confinement dynamique consiste à aspirer et à filtrer l'air au niveau de la source de contamination avec un débit de l'ordre de $3000 \mathrm{~m}^{3} / \mathrm{h}$ (lors de l'ouverture d'une vanne par exemple).

L'utilisation de cette technique permet des gains de temps et de doses, et surtout évite le port de tenues étanches et réduit la quantité de déchets.

\section{La maintenance robotisée}

Pour la réalisation d'opération en milieu hostile où règnent de forts débits de dose, il convient de recourir à des automates permettant d'effectuer les 
manipulations à distance. Le principe consiste à recourir autant que possible à la combinaison de machines-outils simples, conventionnelles, fiables et à des téléopérateurs destinés à visualiser les manipulations par caméra, ainsi qu'à servir la machine-outil (changement de fraise, de foret, de brosse, ramassage d'une pièce tombée...). De tels dispositifs ont été réalisés pour des interventions dans les générateurs de vapeur, sur les tubes guides des internes supérieurs des réacteurs et les plans de joints de pompes primaires. Ces dispositifs complexes font appel à la collaboration des différents services au sein d'EdF (Equipement, Unité technique opérationnelle...) et des constructeurs (Framatome, Inspectronic...). EdF est associé à l'Office de robotique et de productique (OREP) du Commissariat à l'énergie atomique (CEA).

Une étude de remplacement de générateurs de vapeur (GV) est en cours actuellement. L'expérience acquise à l'étranger montre que cette opération est fort coûteuse en dose collective (683 mrem pour deux GV en RFA en 1983). Un outillage et des procédures appropriées sont élaborés actuellement conjointement par EdF et Framatome dans une perspective de réduction maximale des doses prévisibles.

\section{Les actions correctrices, le retour d'expérience}

Les actions correctrices, permettant de réduire la dosimétrie sur les chantiers nucléaires, sont multiples. Elles entrent dans un processus de contrôle et de réduction des doses dans le système de radioprotection. La figure 3 montre, d'une part des moyens de saisie de condition dosimétrique sur les chantiers nucléaires (dosimètres, radiamètres, analyse de la dosimétrie, comptes rendus d'interventions) et, d'autre part, les actions correctrices

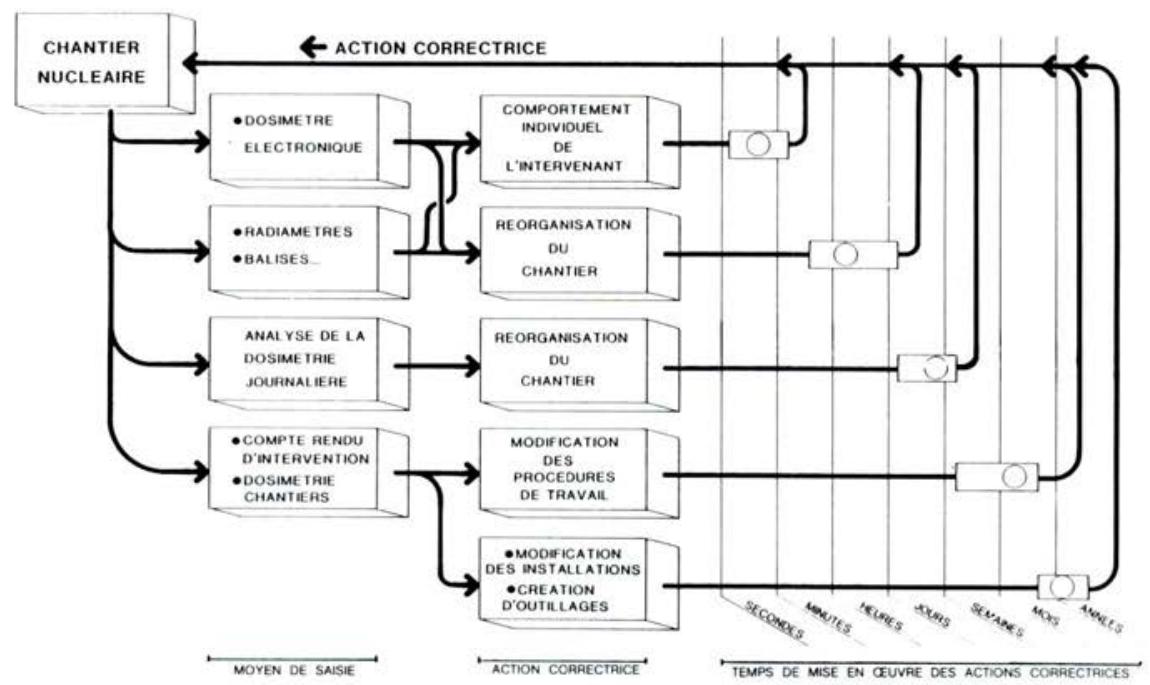

Fig. 3. - Temps de mise en cuvre des actions correctrices en matière de radioprotection. 
correspondantes (comportements individuels, organisation de chantier, modification des procédures de travail et des installations, et création d'outillage). Le temps de mise en œuvre d'actions correctrices est variable. La réaction, à la suite d'une alarme signalant une augmentation locale du débit de dose, est immédiate, tandis que la mise au point d'outillages nouveaux dure plusieurs mois.

Cette multiplicité, cette diversité des actions correctrices et leur imbrication, confère au système de radioprotection la dynamique nécessaire au maintien des doses à un niveau aussi bas que possible.

\section{LA MOTIVATION}

La radioprotection est très bien perçue. Les hommes l'appliquent avec plus de soin que la sécurité classique. Ils portent plus facilement le dosimètre que le casque. On constate au fil des ans une baisse de la dose collective par GWh produit (fig. 4). A l'avenir, le vieillissement des installations et l'importance possible des travaux de maintenance seront de nature à faire croître ces doses. II conviendra de compenser cet effet par une compétence plus grande, basée sur l'expérience acquise.

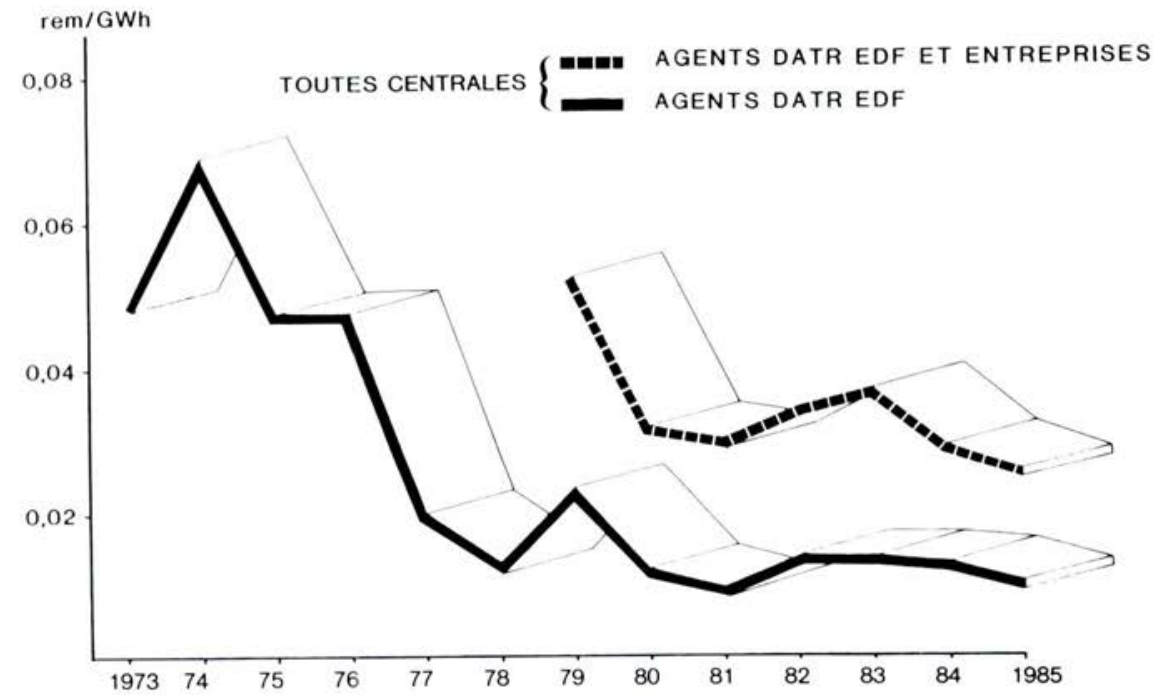

Fig. 4. - Doses collectives rapportées à la production.

A ce jour, après plus de cent années-réacteurs, aucun accident radiologique grave n'est à déplorer en centrales. Les incidents mineurs, qui ont pu avoir lieu, montrent toutefois que la vigilance doit être maintenue.

La maîtrise du risque radiologique s'est développée en même temps que la technique qui l'a généré et il est géré bien avant qu'il ne devienne 
imminent. La radioprotection est un exemple, dans l'histoire technologique, de prise en compte du risque avant une éventuelle catastrophe et non après. Cette gestion implique l'innovation à la fois en matière de technologie et en matière de relations sociales. Cette gestion repose sur les principes, l'organisation et les techniques qui viennent d'être exposés. Mais la réussite de la radioprotection est tributaire en dernier ressort de la motivation des hommes. Celle-ci puise ses ressources au-delà de la technique et de l'organisation.

La motivation des hommes en matière de sécurité et de radioprotection relève d'une adhésion à un projet d'entreprise plus vaste qui inclut la qualité, la sécurité et la sûreté nucléaire. Les stages de formation, les actions de sensibilisation des cadres ainsi que la politique de recrutement sont orientés dans le sens de cette préoccupation essentielle.

Enfin, la motivation individuelle en matière de radioprotection bénéficie d'un mouvement collectif favorable et déterminant au niveau de l'ensemble des acteurs du jeu social: l'opinion publique, le corps médical, l'administration, les partenaires sociaux, ainsi que les milieux industriels et scientifiques.

\section{RÉFÉRENCES}

[1] BERTHET J.J., BERTIN M. Résultats de la dosimétrie du personnel EdF - Enseignements et perspectives. In: SFRP et AIRP. Les expositions professionnelles: aspects méthodologiques et expériences pratiques, Nice, 29-31 janv. 1982. Fontenay-aux-Roses: SFRP, 1986, 235-261.

[2] BOIRON P., CAZAUX J. Le rôle du groupe intersyndical de l'industrie nucléaire (GIIN) dans la protection des travailleurs. In: La radioprotection du personnel des entreprises extérieures intervenant dans les installations nucléaires, Bruxelles, 20-22 mars 1985. Ann. Ass. Belge Radioprot., 1985, 10 (1/2) et Radioprotection, 1986, 21, 64-66.

[3] CAPEL R. L'organisation de la radioprotection du personnel des entreprises extérieures au centre de production nucléaire de Gravelines. In : La radioprotection du personnel des entreprises extérieures intervenant dans les installations nucléaires, Bruxelles, 20-22 mars 1985. Ann. Ass. Belge Radioprot., 1985, 10 (1/ 2) et Radioprotection, 1986, 21, 35-51.

[4] DOLLO R. L'intervention des entreprises extérieures dans les centrales d'Électricité de France. In: La radioprotection du personnel des entreprises extérieures intervenant dans les installations nucléaires, Bruxelles, 20-22 mars 1985. Ann. Ass. Belge Radioprot., 1985, 10 (1/2) et Radioprotection, 1986, 21, 23-34.

[5] EdF, Service Prévention et sécurité, Service de la production thermique. Carnet de prescriptions au personnel radioprotection. Paris: EdF, 1983.

[6] EdF, Service Prévention et sécurité. Radioprotection. Vigilance, 1981, $\mathrm{n}^{\circ} 44$.

[7] EdF, Service de la production thermique, Département Sécurité-radioprotectionenvironnement. Guide pratique de la radioprotection. Paris: EdF, 1985.

[8] EdF, Service de la production thermique, Département Sécurité-radioprotectionenvironnement. Groupe des moyens pédagogiques. Dossiers des stages RP1 et RP2. 
[9] FRANCE. Protection contre les rayonnements ionisants. Paris. Journal officiel de la république française, 1985 (publication $n^{\circ} 1420$ ).

[10] LOCHARD J. Apport de l'évaluation technico-économique dans les choix de radioprotection. In: SFRP et AIRP. Les expositions professionnelles: aspects méthodologiques et expériences pratiques, Nice, 29-31 janv. 1981. Fontenayaux-Roses: SFRP, 1986, 235-261.

[11] ROUSSEL F. Radioprotection du personnel dans les centrales nucléaires. Rev. Gén. Nucl., 1984 (1), 32-37. 